\title{
Cor triatrium dexter
}

\author{
D. Verel, J. Pilcher, and D. M. Hynes \\ From The Cardio-Thoracic Unit, Northern General Hospital, Sheffield $S_{5} 7 A U$
}

\begin{abstract}
A case of cor triatrium dexter is described in which the patient had been independently assessed in two cardiological centres as having a form of Ebstein's anomaly. The differential diognosis is discussed.
\end{abstract}

Septation of the right atrium to form a triatrial heart is a rare anomaly. Most cases have been recorded at necropsy, either as an incidental finding in an otherwise normal heart, or in association with severe congenital heart disease. In most cases the additional partition is due to the persistence of the foetal Eustachian and Thebesian valves which form a septum across the lower part of the atrium. This may vary from a substantial sheet of tissue shown at angiocardiography (Kjellberg et al., 1959) to a reticulum of the type described by Chiari (1897). Cor triatrium has also been used to describe a grossly dilated coronary sinus entering the right atrium by a dilated orifice. Dilatation of this magnitude may be due to the presence of a left superior vena cava, or to anomalous pulmonary veins entering the coronary sinus (Hudson, 1965). Other cardiac defects may be found in association with either defect.

We have been unable to find a clinical account of isolated cor triatrium dexter. Since the differential diagnosis may be of some importance, the following case is reported.

\section{Case report}

The patient was a slightly obese woman aged 21 years. Two years previously heart disease had been diagnosed for the first time when the clinical, radiological, and electrocardiographic findings suggested a mild degree of Ebstein's anomaly. Investigation was delayed by her marriage and an uneventful pregnancy, during which she moved into the Sheffield region. She was referred for advice as she complained of slight dyspnoea on exertion.

On examination she was not dyspnoeic at rest or on moderate exercise. There was no clubbing or central cyanosis. At times there was slight peripheral cyanosis but this appeared to be physiological and due to the cold. The apex beat was normal in character but displaced to the fifth space in the anterior axillary line. The heart sounds were normal, with a soft pansystolic murmur audible from the left sternal edge to the apex.

The electrocardiogram showed a well-marked RSR' pattern most clearly seen in leads II, aVF, and $V_{2}$ to $V_{5}$. The $T$ waves were biphasic in most of these leads.

The outline of the heart on the PA radiograph was enlarged (Fig. Ia), being somewhat like that of Ebstein's anomaly, with clear lung fields. However, the atrial shadow on the right border extended only to the seventh thoracic vertebra. On the lateral and left anterior oblique films, the heart shadow overlay the spine at the level of the tenth rib (Fig. Ib). The shape of this projection was unusual. It was thought initially to be due to an abnormality of the left atrium or ventricle. After angiocardiography, however, it became evident that it was the shadow of the backward projection of the right atrium.

Cardiac catheterization revealed normal intracardiac pressures on the right side of the heart (pulmonary arterial 13/5, right ventricular 13/0, right atrial $3 / 0 \mathrm{~mm} . \mathrm{Hg}$ ). Oximetry did not show any abnormality, arterial saturation being 96 per cent and the venous saturations ranging from 68 per cent in superior vena cava to 75 per cent in inferior vena cava and 73 per cent in main pulmonary artery. Angiocardiography from the main pulmonary artery showed a normal pulmonary artery tree, left atrium, left ventricle, and aorta with no evidence of left-to-right shunt.

Angiocardiographic injection into the superior vena cava (Fig. 2a, b) showed that immediately below the entry of the vena azygos, the superior vena cava widened into a flask-shaped chamber situated behind the main chamber of the right atrium. This flask-shaped chamber extended medially to the left margin of the spine, but did not reach to the lower limit or to the right border of the heart shadow. The inferior vena cava appeared to communicate with the lower extremity of the posterior chamber (Fig. 2b). From this chamber the blood passed forward to fill the an- 


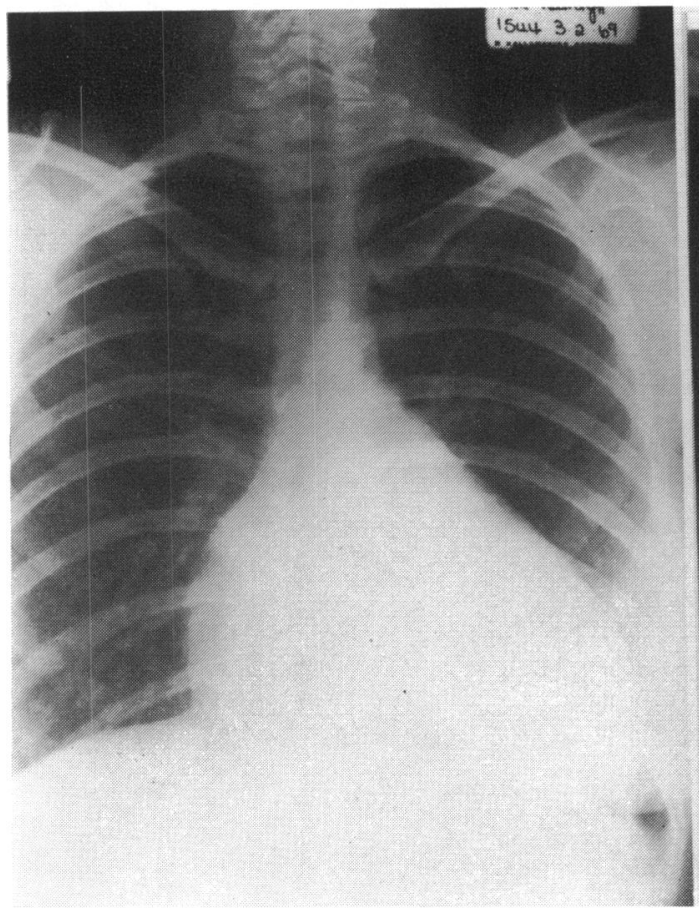

(a)

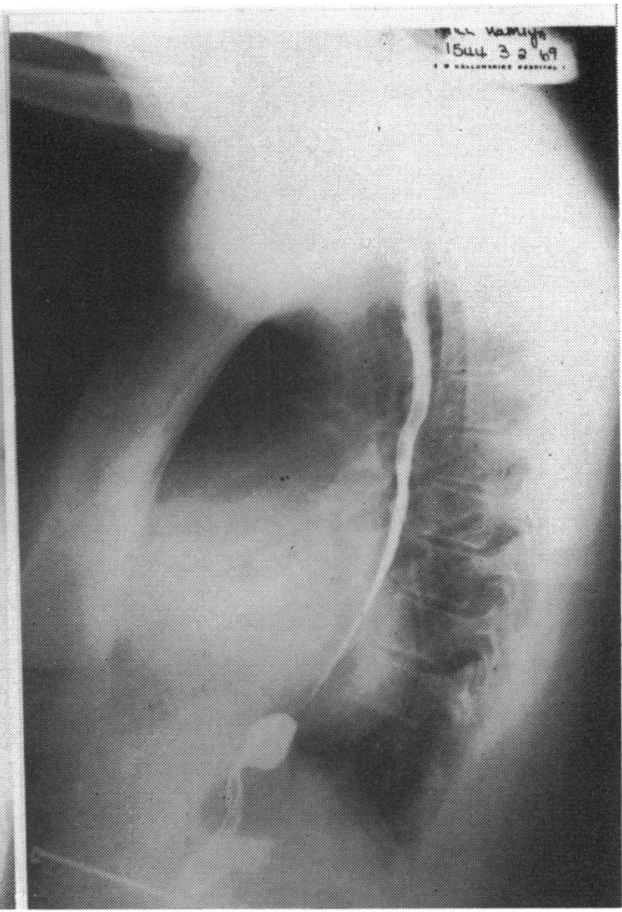

(b)

FIG. I(a) Postero-anterior radiograph of the chest showing globular enlargement of the heart. The outline suggested mild Ebstein's anomaly. (b) Left lateral radiograph of the chest with barium showing apparent enlargement of the left ventricle. This projection of the heart over the shadow of the spine proved to be due to the large right atrium.

terior part of the right atrium (Fig. 2a, b) which formed the right border of the heart shadow. The right ventricle was rotated clockwise by the large atrium and formed the left border of the heart (Fig. 2c). From the right ventricle contrast medium passed through an anatomically normal heart as described above.

The intracardiac electrocardiogram and intracardiac pressure were simultaneously recorded through a $9 \mathrm{~F}$ NIH angiocardiographic catheter using 5 per cent sodium bicarbonate as electrolyte (Stentiford, 1968). A normal change in QRS pattern was recorded at the tricuspid valve, and a normal change in axis of the $P$ wave was found as the catheter was drawn up the right atrium. No significant pressure gradient was found at the area of transition from the main right atrial chamber to the posterior chamber.

\section{Discussion}

The anatomy revealed by cardiac catheterization in this patient appears to correspond to the form of cor triatrium dexter associated with an anomaly of the Eustachian and Thebesian valves. These valves are formed during atrial development when the sinus venosus and parts of the pulmonary veins are incorporated into the atrial walls.
The left horn and transverse portion of the sinus venosus become the oblique vein of the left atrium and coronary sinus, respectively. Simultaneously the atria grow rapidly resulting in the incorporation of the right horn of the sinus venosus into the right atrium. This area is represented in the right atrium by the smooth-walled sinus venarum. The opening of the sinus venosus is initially guarded by a valvular fold. At 3 months the right half of this fold nearly divides the atrium into two chambers. As the heart develops the fold becomes greatly diminished in size. Its cephalic portion becomes the crista terminalis and the caudal portion develops into the folds or valves which guard the inferior vena cava and coronary sinus, i.e. the Eustachian valve. It seems likely that the cor triatrium dexter in this patient is due to a persistence of the whole or part of the primitive valve of the sinus venosus. The not infrequent reports of cases in which this anomaly has been described as an incidental finding at necropsy suggest that it is of no great significance (Yater, 1929). This conclusion is supported by the absence of any pressure gradient between the two atria and by the normal pressures found on 


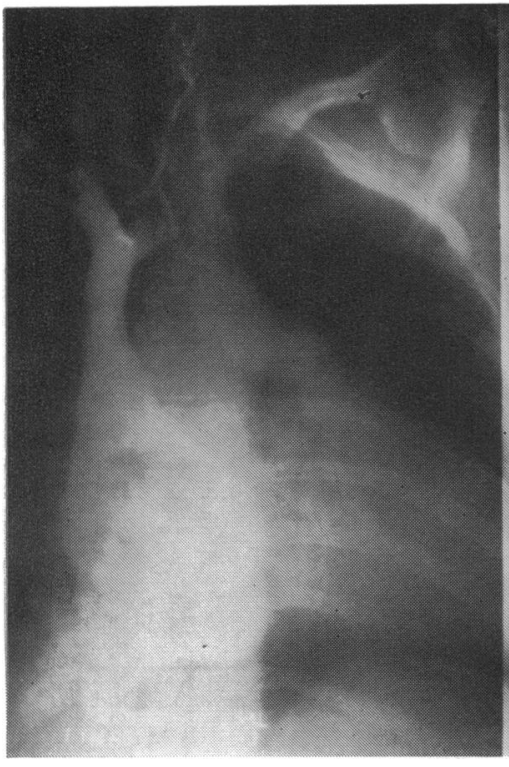

(a)

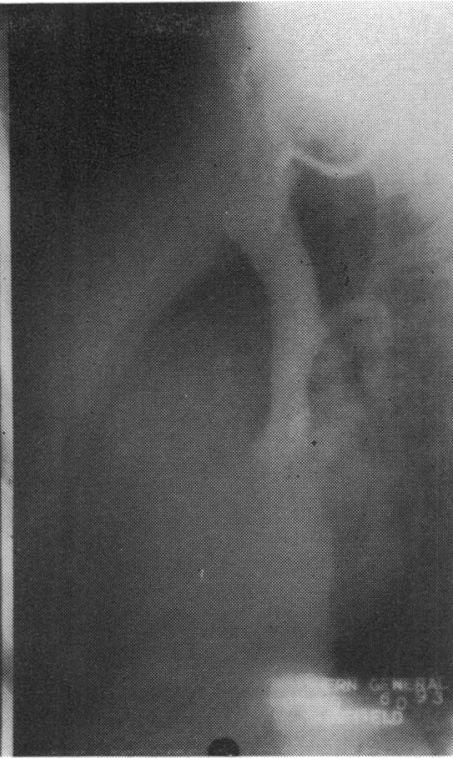

(b)

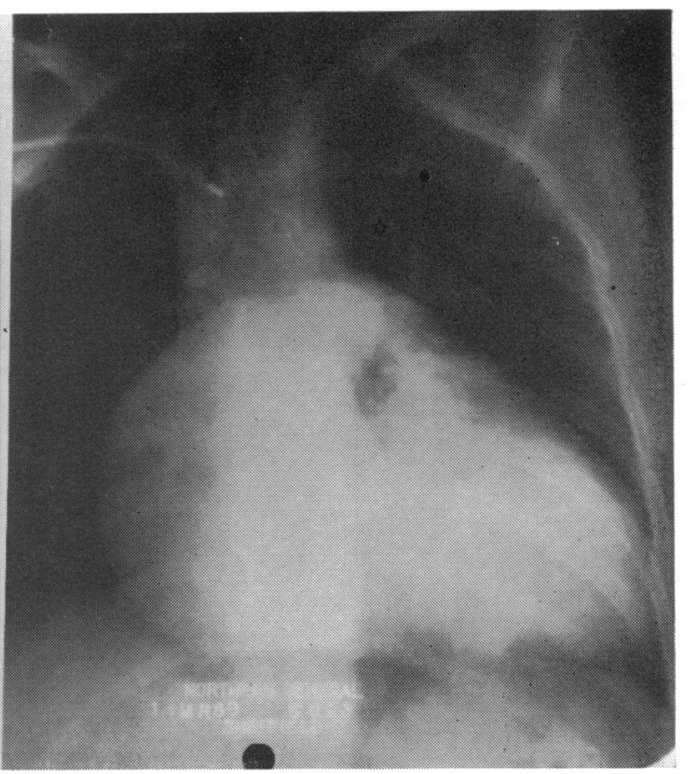

(c)

FIG. 2 (a) and (b) Antero-posterior and lateral films taken one second after injection of contrast medium into the superior vena cava. The posterior chamber of the right atrium is well outlined. The vena azygos can be seen filled retrogradely. The inferior vena cava in the antero-posterior projection can be seen to rise above the lower limit of the anterior atrium to enter the same chamber as the superior vena cava. (c) Postero-anterior film $\mathrm{I}$ second after $\mathrm{a}$ and $\mathrm{b}$ shows a large right atrium and right atrial appendage filled. The right ventricle has opacified. The outline of the superior vena cava and vena azygos is still visible. The right border of the heart is formed by the anterior division of the right atrium, the left border of the heart by the right ventricle.

the right side of the heart and normal left heart anatomy.

This patient was assessed clinically in two regional cardiological centres. In both a diagnosis of Ebstein's anomaly was made on the basis of the physical signs, postero-anterior chest radiograph, and electrocardiogram. The unusual appearance shown on the left lateral and left anterior oblique film, however, suggested that some other lesion was present. In view of the relatively poor prognosis in Ebstein's anomaly and the probable normal outlook in the patient described, this case report appeared justified.

\section{References}

Chiari, H. (1897). Ueber Netzbildungen im rechten Vorhofe des Herzens. Beiträge zur pathologischen anatomie und zur allgemeinen Pathologie, 22, I.

Hudson, R. E. B. (1965). Cardiovascular Pathology. Arnold, London.

Kjellberg, S. R., Mannheimer, E., Rudhe, U., and Jonsson, B. (1959). Diagnosis of Congenital Heart Disease, 2nd ed. Year Book Publishers, Chicago.

Stentiford, N. H. (1968). Increasing the safety of selective angiocardiography. Clinical Radiology, 19, 192.

Yater, W. M. (1929). Variations and anomalies of the venous valves of the right atrium of the human heart. Archives of Pathology, 7, 418. 